before and after onset of the service with a 3- month phasein period, using segmented linear regression with 9-month (April 2014- December 2014) pre- and 36-month (April 2015March 2018) post- intervention periods. Rate of GP interactions were similarly compared before and after using 21month pre- and 36-month post- intervention periods. Rates of A\&E presentations and GP interactions for practice population in Drumchapel- an area within NHS Greater Glasgow \& Clyde with similar rates of deprivation, but with no such service in place - were used as a comparison group. Autoregressive and moving average terms and a fourier term to adjust for seasonality were included in the models.

Results Govan practices had a lower rate of A\&E presentations than Drumchapel practices across all time points. A\&E presentations did not change significantly over time between April and December 2014 in either area. At April 2015, SHIP onset, a level change of $-4.34(-7.44,-1.24)$ A\&E presentations was observed in both areas, but no significant change in trend over time comparing pre and post SHIP in either area. Onset of SHIP was therefore not associated with a reduction in level or trend in A\&E presentations. Rate of interactions with GP was greater for the Govan practices than those of Drumchapel at all time points, prior to January 2015, increasing over time in both areas. After April 2015, there was a significant level change of $33.78(19.57,47.99)$ per 1000 in both areas. GP interactions in Govan however saw a significant reduction of $-1.48(-2.87,-0.09)$ per 1000 per month, following onset of SHIP, between April 2015 and March 2018. This is equivalent to SHIP being associated with an absolute reduction of 37 GP interactions per thousand and a relative reduction of $7.2 \%$ by March 2018 .

Conclusion The Govan SHIP initiative was associated with no significant change in A\&E presentations and a small reduction in GP interactions. A cost effectiveness analysis is recommended.

\section{P34 EVALUATING STAKEHOLDER INVOLVEMENT IN BUILDING A DECISION SUPPORT TOOL FOR NHS HEALTH CHECKS: CO-PRODUCING THE WORKHORSE STUDY}

${ }^{1}$ F Lloyd-Williams*, 'L Hyseni, 'M Guzman-Castillo, ' ${ }^{1} \mathrm{C}$ Kypridemos, 'B Collins, 'S Capewell, ${ }^{1} \mathrm{E}$ Schwaller, ${ }^{2} \mathrm{~A}$ Boland, ${ }^{2} \mathrm{R}$ Dickson, ${ }^{1} \mathrm{M}$ O'Flaherty. ${ }^{1}$ Public Health and Policy, University of Liverpool, Liverpool, UK; ${ }^{2}$ Department of Health Senvices Research, LRiG, University of Liverpool, Liverpool, UK

\subsection{6/jech-2019-SSMabstracts. 185}

Background Ensuring academic research leads to research that is useful for end users is a key challenge in the health research arena. Stakeholder engagement is being increasingly recognised as an important way to achieving impact. The workHORSE project was designed to continuously engage with stakeholders, via four iterative workshops and an e-platform, to inform the development of an open source/open access modelling tool to enable commissioners to quantify the potential cost-effectiveness and equity of the NHS Health Check Programme. An objective of the project is to evaluate the involvement of stakeholders in the process of building the workHORSE computer modelling tool.

Methods The design of the workshop programme was theorybased using the Cairney/Oliver key co-production principles. We identified stakeholders using our extensive networks and snowballing techniques. Iterative development of the decision support modelling tool was informed through engaging with stakeholders during three workshops (to date). We used detailed scripts facilitating open discussion and opportunities for stakeholders to provide additional feedback subsequently. At the end of each workshop, stakeholders completed stakeholder engagement questionnaires to explore their views and experiences throughout the process. The research team also completed questionnaires to explore their expectations prior to the workshops and their experiences thereafter.

Results A total of 25 stakeholders have participated, of which 11 attended two or more workshops. They spanned all levels: local (NHS commissioners, GPs, local authorities and academics), third sector and national organisations (including Public Health England).

Stakeholders experiences were positive overall. They felt valued and commended the involvement of practitioners. Major reasons for attending included being able to influence development and having insight and understanding of what the tool could include and how it would work in practice. They appreciated the iterative process involving a series of workshops which provided opportunities for them to learn about and reflect upon the model's capacity, usage and usefulness. Researchers saw the process as an opportunity for developing a common language and trust in the end product and ensuring the support tool was transparent. The workshops have acted as a reality check ensuring model scenarios and outputs are relevant and fit for purpose.

Conclusion Computational modellers rarely consult with end users when developing tools to inform decision-making. The added value of co-production (collaboration and iteration with stakeholders) potentially enables modellers to produce a 'realworld' operational tool. Likewise, stakeholders have increased confidence in the decision support tool's development and applicability in practice.

\section{P35 USING THE SOCIAL ECOLOGIC FRAMEWORK TO IMPROVE THE INFLUENZA VACCINATION STRATEGY AMONG THE PORTUGUESE ELDERLY}

\begin{abstract}
${ }^{1,2}$ A Machado*, ${ }^{1}$ AC Santos, ${ }^{1,2}$ I Kislay, ${ }^{3} \mathrm{~A}$ Larrauri, ${ }^{1,2} \mathrm{~B}$ Nunes. ${ }^{1}$ Departamento de Epidemiologia, Instituto Nacional de Saude Doutor Ricardo Jorge, Lisbon, Portugal; ${ }^{2}$ Centro de Investigacao Saude Publica, Escola Nacional de Saude Publica, Universidade Nova de Lisboa, Lisbon, Portugal; ${ }^{3}$ CIBER Epidemiologya y Salud Publica (CIBERESP), Instituto de Salud Carlos III, Madrid, Spain
\end{abstract}

\subsection{6/jech-2019-SSMabstracts. 186}

Background In a context of lower than targeted influenza vaccination (IV) coverage, identifying factors associated IV uptake is essential to improve population coverage. Although extensive work has been done in this field, scarcer studies focused on framing these factors within a multifactorial model. Having the social ecological model (SEM) as framework, this study intended to identify and quantify the SEM levels associated to seasonal IV uptake in the Portuguese population aged 65 and more years.

Methods Data from the Portuguese 2014 National Health Survey was restricted to individuals aged 65 and more years $(n=5669)$. The outcome of interest was self-reported IV uptake in the 2 years previous to the interview. Twenty three independent variables were selected from the survey and were allocated in one of the five SEM levels: individual, interpersonal, organizational, community and policy. Sex stratified and age adjusted analysis using Poisson regression were performed 